\title{
DSP Approach to the Design of Nonlinear Optical Devices
}

\author{
Geeta Pasrija \\ Department of Electrical and Computer Engineering, University of Utah, Salt Lake City, UT 84112, USA
}

Email:pasrija@eng.utah.edu

\section{Yan Chen}

Department of Electrical and Computer Engineering, University of Utah, Salt Lake City, UT 84112, USA Email:ychen@ece.utah.edu

\author{
Behrouz Farhang-Boroujeny \\ Department of Electrical and Computer Engineering, University of Utah, Salt Lake City, UT 84112, USA \\ Email: farhang@ece.utah.edu
}

\author{
Steve Blair \\ Department of Electrical and Computer Engineering, University of Utah, Salt Lake City, UT 84112, USA \\ Email:blair@ece.utah.edu
}

Received 5 April 2004; Revised 19 October 2004

\begin{abstract}
Discrete-time signal processing (DSP) tools have been used to analyze numerous optical filter configurations in order to optimize their linear response. In this paper, we propose a DSP approach to design nonlinear optical devices by treating the desired nonlinear response in the weak perturbation limit as a discrete-time filter. Optimized discrete-time filters can be designed and then mapped onto a specific optical architecture to obtain the desired nonlinear response. This approach is systematic and intuitive for the design of nonlinear optical devices. We demonstrate this approach by designing autoregressive (AR) and autoregressive moving average (ARMA) lattice filters to obtain a nonlinear phase shift response.
\end{abstract}

Keywords and phrases: DSP tools, nonlinear optical devices, nonlinear phase shift.

\section{INTRODUCTION}

In order to satisfy the ever-increasing demand for high bit rates, next generation optical communication networks can be made all-optical to overcome the electronic bottleneck and more efficiently utilize the intrinsic broad bandwidth of optical fibers. Currently, there are two possible technologies for achieving high transmission rate: optical time division multiplexing (OTDM) and dense wavelength division multiplexing (DWDM). However, neither the full potential of OTDM nor that of DWDM technology has been realized due to lack of suitable nonlinear, all-optical devices that can perform signal regeneration, ultrafast switching, encoding/decoding, and/or wavelength conversion efficiently.

This is an open access article distributed under the Creative Commons Attribution License, which permits unrestricted use, distribution, and reproduction in any medium, provided the original work is properly cited.
There are a number of problems with current nonlinear optical materials and devices.

There are two types of nonlinear optical materials from which devices can be made: nonresonant and resonant. Nonresonant materials have a weak nonlinear response, but the passage of light occurs with very low loss and the response is broadband, typically exceeding $10 \mathrm{THz}$. However, because of the weak nonlinear response, these devices tend to be bulky and impose a long latency. Resonant materials have a very strong nonlinear response, but at the expense of reduced bandwidths and increased loss. Artificial resonances can be used in optical architectures to overcome the limitations of current nonlinear devices and materials [1]. In this paper, we design nonlinear optical devices that exhibit enhanced nonlinear phase shift response using microring resonators constructed from nonresonant nonlinear material.

The nonlinear optical response of many artificial resonant structures has been studied previously, but most of the 


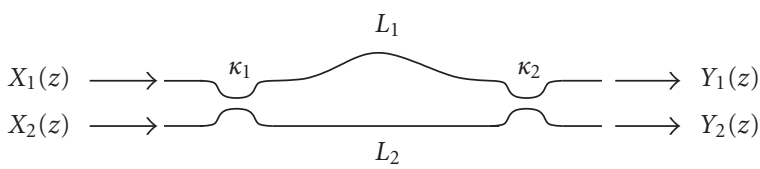

(a)

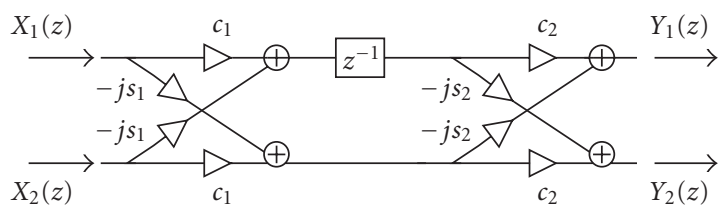

(b)

FIgURE 1: MZI device [2]. (a) Waveguide layout. (b) z-schematic.

studies have been limited to analyzing the nonlinear properties of specific architectures and do not provide a synthesis approach to device design that can produce a specific nonlinear response. Discrete-time signal processing (DSP) provides an easy to use mathematical framework, the z-transform, for the description of discrete-time filters. The z-transform has already been used to analyze numerous optical filter configurations in order to optimize their linear response [2]. We propose a similar approach to optimize the nonlinear response by treating the nonlinear response in the weak perturbation limit as a linear discrete-time filter. The field of discrete-time filter design has been extensively researched and various algorithms are available for designing and optimizing discretetime filters. In this paper, we use existing discrete-time ${ }^{1}$ filter design algorithms to design nonlinear optical devices.

This paper shows that the DSP approach is a systematic and intuitive way to design nonlinear optical devices. Six steps are involved in designing a nonlinear optical device using the DSP approach. First, a prototype linear frequency response (in the weak perturbation limit) is selected for the desired nonlinear optical device. Next, the optical architecture's unit cell is selected and the multistage optical architecture is analyzed using the $\mathrm{z}$-transform. Then, an optimized discrete filter is designed to give the same frequency response as the prototype response desired from the optical architecture in the weak perturbation limit. Next, a mapping algorithm is derived to synthesize the parameters of the optical architecture from the discrete filter. The synthesized optical filter is then simulated using electromagnetic models and its linear response is verified to be the same as that of the discrete filter. Finally, the optical device is simulated to evaluate the desired nonlinear response and confirm the design.

This approach can be used to design optical devices to obtain various nonlinear responses, for example, all-optical switching [3, 4], nonlinear phase shift [5, 6, 7], secondharmonic generation [8], four-wave mixing $[9,10]$ (i.e., frequencies $v_{m}$ and $v_{n}$ mix to produce $2 v_{m}-v_{n}$ and $\left.2 v_{n}-v_{m}\right)$, solitons $[11,12,13]$ (which is a carrier of digital information), bistability $[14,15,16]$ (which results in two stable, switchable output states and can be used as a basis for logic operations and thresholding with restoration), and amplification (which can overcome loss). The nonlinear phase shift is a fundamental nonlinear process that enables many alloptical switching and logic devices, and is the process used to demonstrate our approach. Artificial resonant structures

\footnotetext{
${ }^{1}$ Henceforth, discrete-time filters will be referred to as discrete filters.
}

are used in the devices to overcome the aforementioned traditional drawbacks.

The rest of this paper is organized as follows. Section 2 provides some background on optical filters in relation to discrete-time filters. Section 3 explains the nonlinear phase shift process. Section 4 describes the prototype linear response desired for the nonlinear phase shift. Section 5 discusses the selection of optical architectures. Section 6 details the design procedure for AR and ARMA discrete filters. Sections 7 and 8 outline the mapping of discrete filters on to the optical architectures and their optical response, respectively. Sections 9 and 10 discuss an example and evaluation of AR lattice filters and ARMA lattice filters, respectively, followed by conclusions.

\section{OPTICAL FILTERS AND $z$-TRANSFORMS}

Discrete filters are designed and analyzed using z-transforms. In this section, we discuss the important aspects of optical filters in relation to discrete filters, and explain how ztransforms can be used to describe optical filters as well. This section borrows heavily from Madsen and Zhao's book on optical filters [2]. Like discrete filters, optical filters are completely described by their frequency response. Filters are broadly classified into two categories: finite impulse response (FIR) and infinite impulse response (IIR). FIR filters have no feedback paths between the output and input and their transfer function has only zeros. These are also referred to as moving average (MA) filters. IIR filters have feedback paths and their transfer functions have poles and may or may not have zeros. When zeros are not present or all the zeros occur at the origin, IIR filters are referred as autoregressive (AR) filters. When both poles and nonorigin zeros are present, they are referred to as autoregressive moving average (ARMA) filters.

Optical architectures can be of restricted type or general type. With restricted architectures, we cannot obtain arbitrary frequency response, while general architectures, like discrete filters, allow arbitrary frequency response to be approximated over a frequency range of interest. To approximate any arbitrary function in discrete-time signal processing, a set of sinusoidal functions whose weighted sum yields a Fourier series approximation is used. The optical analog is found in interferometers. Interferometers come in two general classes: (1) Mach-Zehnder interferometer (MZI), and (2) Fabry-Perot interferometer (FPI). MZI is shown in Figure 1a and has finite number of delays and no recirculating (or feedback) delay paths. Therefore, these are MA filters. FPI consists of a cavity surrounded by two partial 


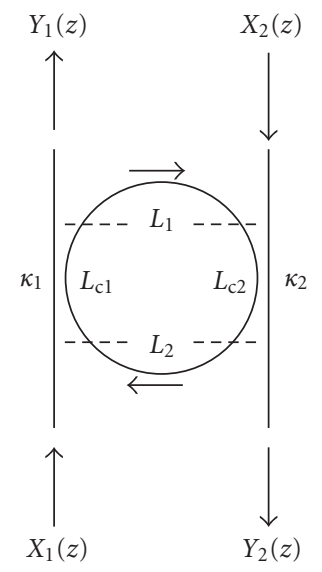

(a)

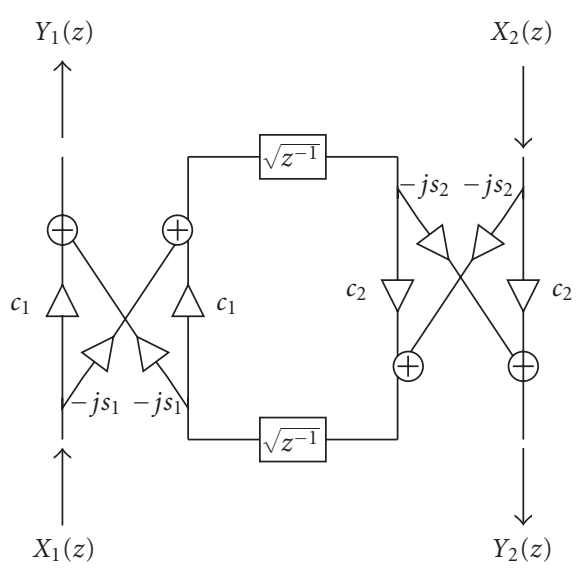

(b)

Figure 2: Ring resonator. After [2]. (a) Waveguide layout. (b) z-schematic.

reflectors that are parallel to each other. The waveguide analog of the FPI is the ring resonator shown in Figure 2a. The output is the sum of delayed versions of the input signal weighted by the roundtrip cavity transmission. The transmission response is of AR type while the reflection response is of ARMA type. The ring resonator is an example of an artificial resonator.

The z-transform schematics for the MZI and FPI device are shown in Figures $1 b$ and $2 b$, respectively. $\kappa$ is the power coupling ratio for each directional coupler, $c=\sqrt{1-\kappa}$ is the through-port transmission term, and $-j s=-j \sqrt{\kappa}$ is the cross-port transmission term. Also, $z=e^{j \Omega T}$, and $\Omega T=\beta \mathrm{E}_{\mathrm{u}}$, where $L_{\mathrm{u}}$ is the smallest path length called the unit delay length, $T$ is the unit delay and is equal to $L_{\mathrm{u}} n / c, \beta$ is a propagation constant and is equal to $2 \pi n / \lambda, n$ is the refractive index of the material, $c$ is the speed of light in vaccum, and $\lambda$ is the wavelength of light. Propagation loss of a delay line is accounted for by multiplying $z^{-1}$ by $\gamma=10^{-\alpha L / 20}$, where $\alpha$ is the average loss per unit length in $\mathrm{dB}$, and $L$ is the delay path length. Because delays are discrete values of the unit delay, the frequency response is periodic. One period is defined as the free spectral range (FSR) and is given by FSR $=1 / T$. The normalized frequency, $f=\omega / 2 \pi$, is related to the optical frequency by $f=\left(\nu-\nu_{\mathrm{c}}\right) T$, or $f=\left(\Omega-\Omega_{\mathrm{c}}\right) T / 2 \pi$. The center frequency $v_{c}=c / \lambda_{c}$ is defined so that the product of refractive index and unit length is equal to an integer number of wavelengths, that is, $m \lambda_{\mathrm{c}}=n L_{\mathrm{u}}$, where $m$ is an integer.

To analyze the frequency response of the MZI, the unit delay is set equal to the difference in path lengths, $L_{\mathrm{u}}=L_{1}-$ $L_{2}$. The overall transfer function matrix of the MZI is the product of the matrices:

$$
\begin{aligned}
\boldsymbol{\Phi}_{\mathrm{MZI}} & =\boldsymbol{\Phi}_{\text {cplr }}\left(\kappa_{2}\right) \boldsymbol{\Phi}_{\text {delay }} \boldsymbol{\Phi}_{\text {cplr }}\left(\kappa_{1}\right) \\
& =\left[\begin{array}{cc}
c_{2} & -j s_{2} \\
-j s_{2} & c_{2}
\end{array}\right]\left[\begin{array}{cc}
z^{-1} & 0 \\
0 & -1
\end{array}\right]\left[\begin{array}{cc}
c_{1} & -j s_{1} \\
-j s_{1} & c_{1}
\end{array}\right] .
\end{aligned}
$$

For the ring resonator, the unit delay is equal to $L_{\mathrm{u}}=$ $L_{1}+L_{2}+L_{\mathrm{c} 1}+L_{\mathrm{c} 2}$, where $L_{\mathrm{c} 1}$ and $L_{\mathrm{c} 2}$ are the coupling region lengths for each coupler. The sum of all-optical paths is given by

$$
Y_{2}(z)=-s_{1} s_{2} \sqrt{\gamma z^{-1}}\left(1+c_{1} c_{2} \gamma z^{-1}+c_{1}^{2} c_{2}^{2} \gamma^{2} z^{-2}+\cdots\right) X_{1}(z) .
$$

The infinite sum simplifies to the following expression for the ring's transfer function:

$$
H_{21}(z)=\frac{Y_{2}(z)}{X_{1}(z)}=\frac{-\sqrt{\kappa_{1} \kappa_{2} \gamma z^{-1}}}{1-c_{1} c_{2} \gamma z^{-1}} .
$$

Other responses for the ring resonator can similarly be obtained. Hence we see that optical resonances are represented by poles in a filter transfer function. Therefore the filters built using artificial resonances are IIR filters.

We have used the MZI and microring resonator as the building blocks to design the nonlinear optical devices for obtaining nonlinear phase shift in this paper. Detailed description of using z-transforms for analyzing single-stage and multistage optical filters is provided in [2].

\section{NONLINEAR OPTICAL PROCESSES}

Nonlinear optics is the study of phenomena that occur as a consequence of the modification of the optical properties of a material under intense illumination. Typically, only laser light is sufficiently intense to modify the optical properties of a material. Nonlinear optical phenomena are nonlinear in the sense that the induced material polarization is nonlinear in the electric field:

$$
\mathbf{P}=\underbrace{\epsilon_{o} \mathbf{E}+\epsilon_{o} \overline{\bar{\chi}}^{(1)}: \mathbf{E}}_{\text {linear } \mathrm{P}^{\mathrm{L}}}+\underbrace{\epsilon_{o} \overline{\bar{\chi}}^{(2)}:: \mathbf{E} \cdot \mathbf{E}+\epsilon_{o} \overline{\overline{\bar{\chi}}}^{(3)}:: \mathbf{E} \cdot \mathbf{E} \cdot \mathbf{E}+\cdots}_{\text {nonlinear } \mathrm{P}^{\mathrm{NL}}},
$$

where dielectric dispersion is ignored. The optical Kerr effect (i.e., nonlinear refraction index) results from the third-order nonlinear susceptibility $\overline{\overline{\bar{\chi}}}^{(3)}$, which is a fourth-rank tensor.

An optical wave is a real quantity and is usually expressed as

$$
\mathbf{E}(t)=\operatorname{Re}\{\mathbf{E} \exp j(\mathbf{k} \cdot \mathbf{r}+\omega t)\},
$$


or similarly as

$$
\mathbf{E}(t)=\frac{1}{2} \mathbf{E} \exp j(\mathbf{k} \cdot \mathbf{r}+\omega t)+c \mathbf{c},
$$

where cc represents the complex conjugate of the preceding term. Thus, an $\mathrm{x}$-polarized optical wave, propagating in $\mathrm{z}$ direction in an isotropic medium, is represented mathematically as

$$
\mathbf{E}(t)=\frac{1}{2} E_{x} \hat{x} \exp j(k z+\omega t)+\mathrm{cc} .
$$

\subsection{Nonlinear phase shift}

The third-order polarization (mediated by $\chi^{(3)}$ ) in a material leads to a nonlinear intensity dependent contribution to its refractive index, that is, the refractive index of the material changes as the incident intensity on the material changes. The susceptibility tensors in isotropic material can be further simplified as $\overline{\bar{\chi}}^{(1)}=\chi^{(1)}$, being a scalar quantity, and $\overline{\bar{\chi}}^{(2)}=0$, due to inversion symmetry. The third-order nonlinear susceptibility will only have one contributing term $\chi_{x x x x}$ since the light is $\mathrm{x}$-polarized and there are no means for sourcing additional polarization components. The linearand nonlinear-induced polarizations are

$$
\begin{aligned}
P^{\mathrm{L}}= & \epsilon_{o}\left(1+\chi^{(1)}\right) E, \\
P^{\mathrm{NL}}= & P^{(3)} \\
= & \epsilon_{o} \chi_{x x x x}(\omega ;-\omega, \omega, \omega) E^{*} E E \\
& +\epsilon_{o} \chi_{x x x x}(\omega ; \omega,-\omega, \omega) E E^{*} E \\
& +\epsilon_{o} \chi_{x x x x}(\omega ; \omega, \omega,-\omega) E E E^{*} \\
= & 3 \epsilon_{o} \chi_{x x x x}|E|^{2} E \\
= & \frac{3}{4} \epsilon_{o} \chi_{x x x x}\left|E_{x}\right|^{2} E,
\end{aligned}
$$

respectively. Hence,

$$
P=P^{\mathrm{L}}+P^{\mathrm{NL}}=\epsilon_{o}\left(1+\chi^{(1)}+\frac{3}{4} \epsilon_{o} \chi_{x x x x}\left|E_{x}\right|^{2}\right) E .
$$

The total dielectric constant is

$$
\epsilon_{r}^{\mathrm{tot}}=\epsilon_{r}+\Delta \epsilon_{r}
$$

Comparing with the expression for $P$, we obtain $\epsilon_{r}=1+$ $\chi^{(1)}=n_{o}^{2}$ and $\Delta \epsilon=(3 / 4) \chi_{x x x x}\left|E_{x}\right|^{2}$. The refractive index is related to the dielectric constant as

$$
n=\sqrt{\epsilon_{r}+\Delta \epsilon_{r}} \approx \sqrt{\epsilon_{r}}+\frac{\Delta \epsilon_{r}}{2 \sqrt{\epsilon_{r}}}=n_{o}+\frac{3 \chi_{x x x x}}{8 n_{o}}\left|E_{x}\right|^{2} .
$$

The intensity dependent refractive index for a nonlinear material is given by

$$
n=n_{o}+n_{2}|E|^{2}
$$

Comparing (11) and (12), the nonlinear refractive index is directly determined by the third-order susceptibility as

$$
n_{2}=\frac{3 \chi_{x x x x}}{8 n_{o}}=\frac{3 \chi^{(3)}}{8 n_{o}}
$$

which characterizes the strength of the optical nonlinearity. The intensity $I$ of an optical wave is proportional to $|E|^{2}$ as $I=(1 / 2 \eta)|E|^{2}$ where $\eta$ is the impedance of the medium. When comparing the optical response in the same medium, $I=|E|^{2}$ is taken for simplification.

This intensity dependent refractive index, in turn, results in various processes, one of which is the nonlinear phase shift. For a material with positive $n_{2}$, increasing the intensity results in a red shift of the frequency response of an optical filter. This can be explained using the equation $n L_{\mathrm{u}}=m \lambda_{\mathrm{c}} \Rightarrow$ $\left(n_{o}+n_{2}|E|^{2}\right) L_{\mathrm{u}}=m \lambda_{\mathrm{c}}$, where $m$ is an integer. The product $n L_{\mathrm{u}}$ is called the optical path length. Increasing intensity $I$ results in the increase of optical path length and wavelength $\lambda_{c}$, and hence a decrease in the center frequency $\nu_{c}$ causing a red shift of the frequency response. When optical path length is increased by varying $L_{\mathrm{u}}$ and keeping $n$ constant, the red shift will be perfect and the shape of the frequency response curve will not change. In nonlinear materials, the refractive index $n$ as well as the loss in the material changes with changing intensity and hence the red shift is not perfect.

As discussed, current nonlinear optical materials and devices either have weak nonlinear response (nonresonant materials) or have high loss (resonant materials). Using artificial resonances, for example, microring resonators made of nonresonant nonlinear material, we can obtain strong nonlinear response with low loss [1]. Light circulates within the resonator and coherent interference of multiple beams occurs, resulting in intracavity intensity build-up and group delay enhancement which in turn enhances the nonlinear response.

\section{PROTOTYPE RESPONSE FOR NONLINEAR PHASE SHIFT}

The nonlinear phase shift is a fundamental nonlinear process that enables many all-optical switching and logic devices [5] that can be used in the next generation optical communication systems. An ideal nonlinear phase shifting element has constant intensity transmission up to at least a $\pi$ radian phase shift upon increasing the incident intensity. The lesser the intensity required to obtain a $\pi$ phase shift, the better the nonlinear performance.

The first step in the design approach is to select a linear frequency response for the desired device. Figure 3 illustrates the notion of producing a nonlinear phase shift response through the nonlinear detuning of a periodic (discrete) filter. To act as an ideal nonlinear phase shifter, in the weak perturbation limit, a flat magnitude response and steep linear phase are desired within the passband.

Light incident on the filter (at a frequency $v_{m}$, e.g.) will be transmitted with efficiency given by the magnitude response, but will also experience a phase change due to the 

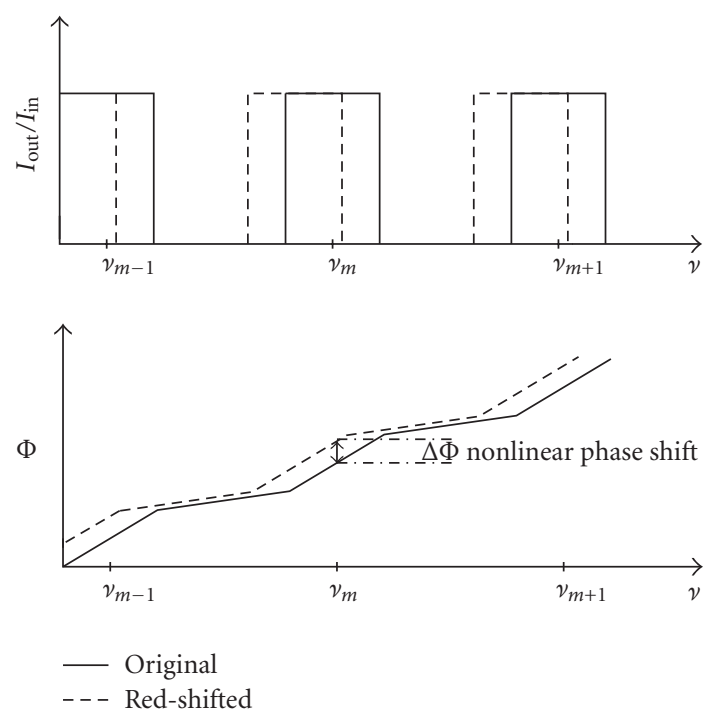

FIGURE 3: Prototype linear response for nonlinear phase shift.

phase response. As the light intensity increases, the overall filter response will red shift due to intensity-induced changes in the filter components, which are themselves constructed from (weakly) nonlinear materials. Ideally, under weak detuning, the transmitted intensity fraction will not change (and hence the desire for a flat-topped magnitude response), but the phase at the output will change due to a steep linear phase response within the filter passband. The slope of the phase determines the group delay. Ripples in group delay may result in bistability in the nonlinear response, and therefore, linear phase is desired in the passband to have constant group delay. In effect, what this approach does is to amplify the intrinsic nonlinearity of a material, where the efficiency of the process improves with increasing the filter group delay. However, strong detuning in multiresonator systems can result in distortions of the filter response.

The red-shifted response is shown by the dotted curve in Figure 3. It can be seen that the transmitted output does not change (in the weak perturbation limit) and a nonlinear phase shift is obtained because of the shifted phase response. An increase in the input intensity $I_{\text {in }}$ results in greater red shift and hence more nonlinear phase shift. The input intensity at which a $\pi$ phase shift is obtained is denoted as $I_{\pi}$. The nonlinear phase shift response should be such that a phase shift of $\pi$ can be obtained at a lower input intensity, $I_{\pi}$, than that required for the bulk material. The lower the $I_{\pi}$, the better the filter. Also, the transmission ratio at the intensity at which $\pi$ phase shift is obtained should be at least 0.5 , for maximum of $3 \mathrm{~dB}$ insertion loss.

\section{OPTICAL ARCHITECTURES FOR NONLINEAR PHASE SHIFTER}

The second step is to select the optical architecture's unit cell and analyze it using the z-transform. Artificial resonances produced by ring resonators can be used to enhance the nonlinear phase shift response of an optical device $[1,7]$.

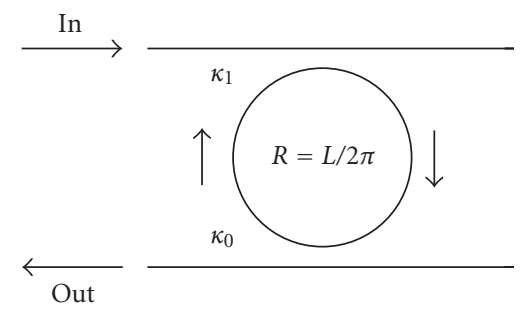

FIgURE 4: Single-pole structure.

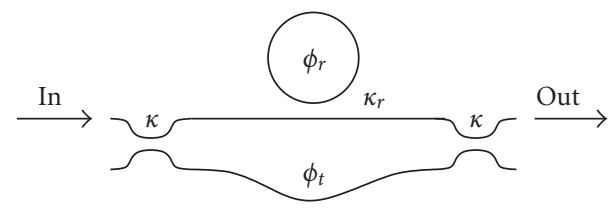

FIgURE 5: Independent pole-zero structure.

The presence of a ring resonator in the architecture implies the presence of a pole in the filter's transfer function. To select the optical architecture for obtaining a nonlinear phase shift response, we analyze two ring resonator configurations (1) single pole (2) single pole-zero with the pole and zero independent of each other.

(i) Single-pole design. Figure 4 shows a single-pole architecture with a zero at the origin. The transfer function for this architecture in the $\mathrm{z}$-domain is given by

$$
\frac{E_{\text {out }}(z)}{E_{\text {in }}(z)}=\frac{\sqrt{\kappa_{0} \kappa_{1}} \sqrt{\gamma e^{-j \phi} z^{-1}}}{1-c_{0} c_{1} \gamma e^{-j \phi} z^{-1}}
$$

The total phase change in the fundamental range $-\pi \leq$ $\omega \leq \pi$ for this unit cell is equal to $\pi$. By cascading $N$ such unit cells, we can obtain a total phase change of $N \pi$ in the fundamental range.

(ii) Single pole-zero design with independent pole and zero. Figure 5 shows a single pole-zero architecture with the pole and zero independent of each other. The transfer function for this architecture in the $\mathrm{z}$-domain is given by

$$
\frac{E_{\text {out }}(z)}{E_{\text {in }}(z)}=\frac{\left(c^{2} c_{r}-s^{2} e^{-j \phi_{t}}\right)-\left(c^{2} e^{-j \phi_{r}}-s^{2} c_{r} e^{-j\left(\phi_{r}+\phi_{t}\right)}\right) z^{-1}}{1-c_{r} e^{-j \phi_{r}} z^{-1}} .
$$

The total phase change in the fundamental range $-\pi \leq$ $\omega \leq \pi$ for this unit cell is equal to $2 \pi$ if the filter is maximum phase, and 0 if it is minimum phase. We are interested in lowpass maximum phase systems ( $\mid$ zero $|>1 /|$ pole $\mid$ ) since they have the maximum net phase change and most of the phase change lies within the passband. The architecture shown in Figure 5 can be designed to be a lowpass maximum phase system since the poles and zeros are independent of each other. By cascading $N$ such unit cells, we can obtain a total phase change of $2 N \pi$ in the fundamental range. 


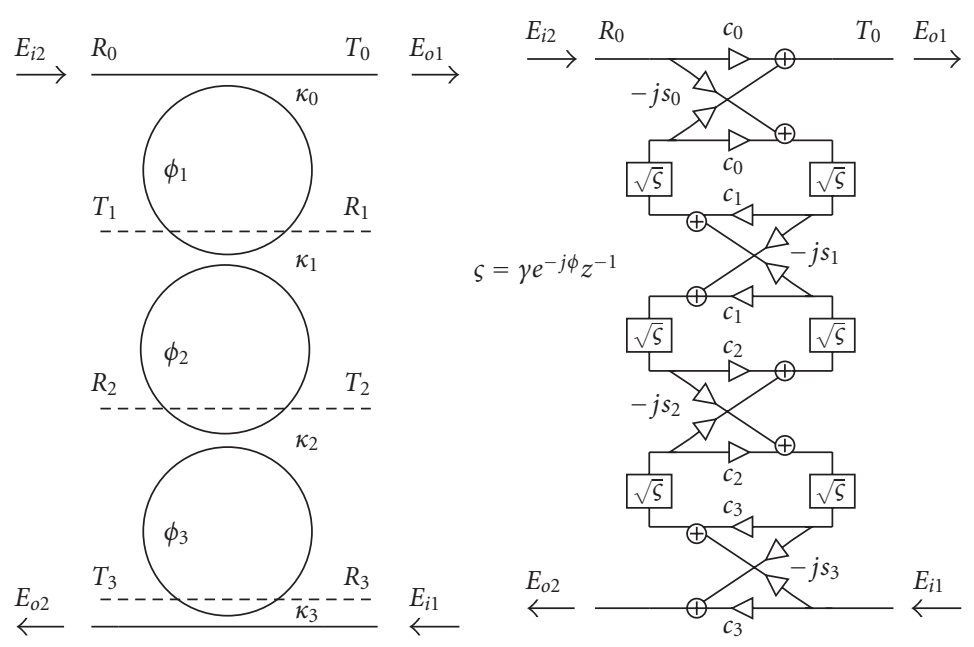

Figure 6: AR lattice filter [2].

A third possible configuration is a ring resonator with a single coupler. However, this is a pole-zero architecture with dependent pole and zero and is always highpass for a maximum phase system. The total phase change is equal to $2 \pi$ but most of the phase change is present in the stopband and hence, we cannot obtain the prototype response of Figure 3 using this unit cell. Therefore, we decided to use the first and second configurations as the unit cells for our designs. Joining the first configuration unit cell in a lattice structure gives us an AR lattice filter architecture shown in Figure 6 and joining the second configuration unit cell in a lattice structure gives us an ARMA lattice filter architecture shown in Figure 7. Lattice structures are chosen since they have low passband loss and can operate at significantly higher component variations as compared to transversal or cascade structures.

The next step is to obtain a z-transform description of the multistage architecture obtained by joining the unit cells. First, a DSP schematic is drawn for the architecture and then it is analyzed to obtain a transfer function matrix. The AR and ARMA lattice architecture's DSP schematics and transfer functions are given below. The detailed derivations are presented in [2].

(i) AR lattice filter. Figure 6 shows the waveguide layout and DSP schematic of an AR lattice architecture. The transfer matrix for this architecture is [2]

$$
\left[\begin{array}{c}
T_{n+1}(z) \\
R_{n+1}(z)
\end{array}\right]=\boldsymbol{\Phi}_{N} \boldsymbol{\Phi}_{N-1} \cdots \boldsymbol{\Phi}_{1} \boldsymbol{\Phi}_{0}\left[\begin{array}{c}
T_{0}(z) \\
R_{0}(z)
\end{array}\right]
$$

where

$$
\Phi_{n}=\frac{1}{-j s_{n} \sqrt{\gamma e^{-j \phi_{n+1}} z^{-1}}}\left[\begin{array}{cc}
1 & -c_{n} \\
c_{n} \gamma e^{-j \phi_{n+1}} z^{-1} & -\gamma e^{-j \phi_{n+1}} z^{-1}
\end{array}\right]
$$

(ii) ARMA lattice filter. Figure 7 shows the waveguide layout and DSP schematic of an ARMA lattice architecture. The transfer matrix for this architecture is [2]

$$
\left[\begin{array}{c}
X_{n}(z) \\
Y_{n}(z)
\end{array}\right]=\boldsymbol{\Phi}_{N} \boldsymbol{\Phi}_{N-1} \cdots \boldsymbol{\Phi}_{1} \boldsymbol{\Phi}_{0}\left[\begin{array}{c}
X_{0}(z) \\
Y_{0}(z)
\end{array}\right]
$$

where

$$
\begin{aligned}
& \boldsymbol{\Phi}_{n}=\frac{1}{A_{n}(z)}\left[\begin{array}{cc}
-c_{n t} A_{n}^{R}(z) e^{-j \phi_{n r}} & -j s_{n t} A_{n}(z) e^{-j \phi_{n t}} \\
j s_{n t} A_{n}^{R}(z) e^{-j \phi_{n r}} & c_{n t} A_{n}(z) e^{-j \phi_{n t}}
\end{array}\right], \\
& A_{n}=1-c_{n r} e^{-j \phi_{n r}} z^{-1}, \quad A_{n}^{R}=-c_{n r}+e^{-j \phi_{n r}} z^{-1} .
\end{aligned}
$$

\section{DESIGN OF ARMA AND AR DISCRETE FILTERS}

The next step is to design discrete filters to be mapped onto AR and ARMA lattice architectures with the response as shown in Figure 3 (where the number of stages, i.e., poles and zeros are given). For mapping onto the AR lattice architecture having $N$ rings (unit cells), an $N$ th-order discrete AR filter ( $N$ poles, no zeros) is designed. Similarly, for mapping onto the ARMA lattice architecture having $N$ stages, an $N$ th-order discrete ARMA filter ( $N$ poles and $N$ zeros) is designed. The discrete filter design procedure for designing AR and ARMA filters is described below. The design needs to meet the constraints of linear phase within the passband with as high group delay as possible, and flat magnitude response with as large bandwidth as possible.

\subsection{Design of AR discrete filters}

Each stage of the AR optical architecture represents a pole in the transfer function. Therefore, the discrete filter designed to be mapped on this architecture should have only poles. To obtain the nonlinear phase shift, the AR discrete filter should be designed to obtain the prototype response 


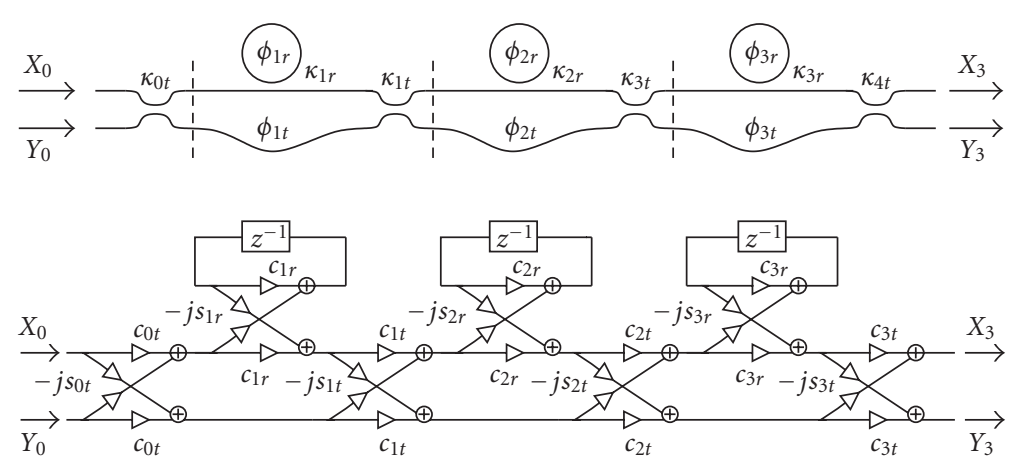

FIgURE 7: ARMA lattice filter [2].

of Figure 3. The prototype response requires a flat passband and linear phase within the passband. If $H(z)$ is the transfer function of the discrete filter, the condition to obtain linear phase is $H\left(z^{-1}\right)=z^{-\Delta} H(z)$, where $\Delta$ is a delay. In the case of IIR filters, since all poles are inside the unit circle, satisfying the above condition requires that there are mirror image poles outside the unit circle thereby making the filter unstable. Therefore, stable IIR filters can only approximate a linear phase response.

In the next subsection, we formulate the problem of ARMA discrete filter design as a least squares minimization problem. Since the case of AR filters can be thought as a special case of ARMA filters with all zeros at origin, the least squares formulation of ARMA filter design can be easily adopted to AR filters as well. However, unfortunately, numerical examples reveal that this approach results in either unstable IIR filters or, if the poles of the filter are constrained to the stable region, $|z|<1$, the group delay of the resulting filter will be unsatisfactory. Therefore, other methods of filter design have to be adopted. Selesnick and Burrus [17] have proposed a generalized Butterworth discrete filter design procedure that allows arbitrary constraints to be imposed on the number of poles and nontrivial zeros, that is, zeros other than those at the origin. Hence, it can be adopted for designing AR filters. The designs satisfy the condition of maximally flat magnitude response at the center of passband, the Butterworth condition. This fulfills the required flat passband response. The filter's group delay shows some variation over the passband. However, it remains relatively flat over a good portion of the passband, which, to some extent, satisfies the constant group delay condition.

The generalized Butterworth filter design uses the mapping $x=(1 / 2)(1-\cos (\omega))$ and provides formulas for two real and nonnegative polynomials $P(x)$ and $Q(x)$ where $P(x) / Q(x)$ resembles a lowpass response, over the range $x \in$ $[0,1]$ (equivalent to $\omega \in[0, \pi]$ ). A stable IIR filter $B(z) / A(z)$ that satisfies

$$
\left|H\left(e^{j \omega}\right)\right|^{2}=\frac{P(1 / 2-(1 / 2) \cos \omega)}{Q(1 / 2-(1 / 2) \cos \omega)}
$$

is then obtained. To this end, the spectral factorizations $P(1 / 2-(1 / 2) \cos \omega)=B\left(e^{j \omega}\right) B\left(e^{-j \omega}\right)$ and $Q(1 / 2-$
$(1 / 2) \cos \omega)=A\left(e^{j \omega}\right) A\left(e^{-j \omega}\right)$, from which the transfer functions $B(z)$ and $A(z)$ could be extracted, are performed. Note that the latter factorizations are possible since $P(x)$ and $Q(x)$, for $x \in[0,1]$, are real and nonnegative [18].

Reference [17] details the design process and provides the closed form expressions for obtaining $B(z)$ and $A(z)$. The routine maxflat provided in the Matlab's signal processing toolbox is an implementation of the generalized Butterworth filter design procedure. We use this routine of Matlab to design the AR filters whose response matches the prototype response. The number of poles and the bandwidth are given as parameters to the routine which delivers the desired transfer function.

\subsection{Design of ARMA discrete filters}

The generalized Butterworth filter design procedure that was considered above for the design of AR filters could also be adopted for the design of ARMA filters. However, our experiments have shown that better designs could be obtained by adopting a least squares method. The idea is to find the coefficients of an IIR transfer function

$$
H(z)=\frac{B(z)}{A(z)}=\frac{b_{0}+b_{1} z^{-1}+\cdots+b_{N} z^{-N}}{1+a_{1} z^{-1}+\cdots+a_{N} z^{-N}}
$$

such that its frequency response resembles that of a desired response. Two approaches are commonly adopted [19]: (i) the output error method, and (ii) the equation error method. In the output error method, the coefficients of $A(z)$ and $B(z)$ are chosen by minimizing the cost function

$$
\xi_{\mathrm{oe}}=\frac{1}{2 \pi} \int_{0}^{2 \pi} W(\omega)\left|\frac{B\left(e^{j \omega}\right)}{A\left(e^{j \omega}\right)}-H_{o}\left(e^{j \omega}\right)\right|^{2} d \omega
$$

where $W(\omega)$ is a weighting function and $H_{o}\left(e^{j \omega}\right)$ is the desired (prototype filter) response. In the equation error method, on the other hand, the coefficients of $A(z)$ and $B(z)$ are chosen by minimizing the cost function

$$
\xi_{\mathrm{ee}}=\frac{1}{2 \pi} \int_{0}^{2 \pi} W(\omega)\left|B\left(e^{j \omega}\right)-A\left(e^{j \omega}\right) H_{o}\left(e^{j \omega}\right)\right|^{2} d \omega .
$$


In this paper, we choose the equation error method as it leads to a closed form solution for the filter coefficients. The output error method leads to a nonlinear optimization procedure. It is thus much harder to solve. Moreover, any solution that could be obtained from the output error method may also be obtained from the equation error method by an appropriate selection of the weighting function $W(\omega)$.

The common approach of optimizing $B\left(e^{j \omega}\right)$ and $A\left(e^{j \omega}\right)$ in (24) is to first replace the integral (24) by the weighted sum

$$
J_{\mathrm{ee}}=\sum_{i=1}^{K} w_{i}\left|B\left(e^{j \omega_{i}}\right)-A\left(e^{j \omega_{i}}\right) h_{o, i}\right|^{2},
$$

where $\omega_{i}$ is a grid of dense frequencies over the range $0 \leq \omega \leq$ $2 \pi$ and $w_{i}$ is the short-hand notation for $W\left(\omega_{i}\right)$. Defining the column vectors

$$
\begin{aligned}
\mathbf{e}_{i}=[ & 1 e^{j \omega_{i}} e^{j 2 \omega_{i}} \cdots e^{j N \omega_{i}}-h_{o, i} e^{j \omega_{i}}-h_{o, i} e^{j 2 \omega_{i}} \\
& \left.\cdots-h_{o, i} e^{j N \omega_{i}}\right]^{H},
\end{aligned}
$$

$\mathbf{b}=\left[\begin{array}{lllll}b_{0} & b_{1} & b_{2} & \cdots & b_{N}\end{array}\right]^{H}, \mathbf{a}=\left[\begin{array}{llll}a_{1} & a_{2} & \cdots & a_{N}\end{array}\right]^{H}$, where the superscript $H$ denotes Hermitian, and $\mathbf{c}=\left[\begin{array}{l}\mathbf{b} \\ \mathbf{a}\end{array}\right],(25)$ can be rearranged as

$$
J_{\mathrm{ee}}=\mathbf{c}^{H} \boldsymbol{\Psi} \mathbf{c}-\boldsymbol{\theta}^{H} \mathbf{c}-\mathbf{c}^{H} \boldsymbol{\theta}+\eta,
$$

where

$$
\begin{gathered}
\boldsymbol{\Psi}=\sum_{i=1}^{K} w_{i} \mathbf{e}_{i} \mathbf{e}_{i}^{H}, \\
\boldsymbol{\theta}=\sum_{i=1}^{K} w_{i} h_{o, i} \mathbf{e}_{i},
\end{gathered}
$$

and $\eta=\sum_{i=1}^{K} w_{i}\left|h_{o, i}\right|^{2}$.

The cost function (27) has a quadratic form whose solution is well known to be [19]

$$
\mathbf{c}=\Psi^{-1} \boldsymbol{\theta}
$$

Once $\mathrm{c}$ is obtained, one can easily extract the coefficients $b_{i}$ and $a_{i}$ from it. This procedure was originally developed in [20].

The routine invfreqz in Matlab signal processing tool box can be used to find the coefficients $A(z)$ and $B(z)$ according to the above procedure.

\section{MAPPING DISCRETE FILTERS ONTO OPTICAL ARCHITECTURES}

The optical architectures were analyzed using the $\mathrm{z}$ transform and their transfer functions were derived in Section 5. The discrete filter's transfer functions obtained in the previous step are now set equal to the corresponding optical filter's transfer function. Backward relations are derived to calculate the optical architecture's parameters for each stage. Thus, the optical filter is synthesized from the discrete filter using a mapping algorithm. The AR discrete filter designed in the previous section is mapped onto the AR lattice optical architecture using the recursion-based algorithm developed by Madsen and Zhao [21]. The ARMA discrete filter designed in the previous section is mapped onto the ARMA lattice optical architecture using the recursion-based algorithm developed by Jinguji [22]. These algorithms return the coupling ratios and phase solutions for each stage of the lattice architectures.

\section{FROM DISCRETE RESPONSE TO THE OPTICAL RESPONSE}

The optical filter designed using the above steps is now simulated for its linear response [23] using electromagnetic models. Also, the linear optical response is compared with the discrete filter's response. Both should have exactly the same shape (different scales) since the optical filter was synthesized from the discrete filter.

The discrete frequency response curve can be converted to an optical frequency response curve once we know the optical parameters such as unit length and center frequency. We had previously defined $z=e^{j \Omega T}$ with $\Omega=2 \pi v$, and $T=L_{\mathrm{u}} n / c$ where $v$ is the optical frequency, $L_{\mathrm{u}}$ is the unit length, $n$ is the refractive index, and $c$ is the speed of light. Also the FSR was defined to be equal to $1 / T$.

The discrete frequency response plotted over the fundamental range $-\pi \leq \omega \leq \pi$ or $-1 / 2 \leq f \leq 1 / 2$ which is normalized to $-1 \leq f_{\text {norm }} \leq 1$ by Matlab's freqz routine is equal to one optical FSR. The normalized frequency $f_{\text {norm }}=\omega_{\text {norm }} / 2 \pi$ is related to the optical frequency by $f_{\text {norm }}=\left(\nu-\nu_{\mathrm{c}}\right) T$ or $f_{\text {norm }}=\left(\Omega-\Omega_{\mathrm{c}}\right) T / 2 \pi$. To plot the optical frequency response over one FSR directly using freqz, the sampling frequency $F_{s}$ can be set equal to the FSR and the frequency response can be plotted from $-F_{s} / 2$ to $F_{s} / 2$.

Since FSR $=1 / T=c / n L_{\mathrm{u}}$, we need to know the unit length to know FSR. The unit length is chosen such that the product of refractive index and unit length is equal to an integer number of wavelengths, that is, $m \lambda_{\mathrm{c}}=n L_{\mathrm{u}}$ where $m$ is an integer and $\lambda_{c}$ is the desired central wavelength. The center frequency is then defined as $\nu_{c}=c / \lambda_{c}$. It is the frequency at which resonance occurs.

Once the linear response of the optical architecture is verified to be the same as that of the discrete filter, the optical filter is simulated to obtain the nonlinear phase shift response [23].

\section{EXAMPLE AND EVALUATION OF AR LATTICE FILTERS}

\subsection{Design and synthesis example}

In this section, we design an optical AR lattice filter and simulate it to obtain the nonlinear phase shift response. The filter is synthesized by designing discrete filters according to the 
description in Section 6.1 and then using the mapping algorithm derived by Madsen and Zhao [21]. The circumference of each microring in the AR lattice architecture is chosen as the unit delay length and is equal to $50 \mu \mathrm{m}$. The center frequency corresponds to a wavelength of $500 \mathrm{~nm}$.
A generalized digital Butterworth filter with five poles is designed using the procedure discussed in Section 6.1. Filter bandwidth is set to be $0.16 \pi$ in the fundamental range $-\pi \leq$ $\omega \leq \pi$. Assuming the loss in the material to be $1 \mathrm{~cm}^{-1}$, the obtained filter transfer function is

$$
\frac{N(z)}{D(z)}=\frac{6.5941 \times 10^{-4}}{1.0000-4.1912 z^{-1}+7.0824 z^{-2}-6.0254 z^{-3}+2.5789 z^{-4}-0.4439 z^{-5}}
$$
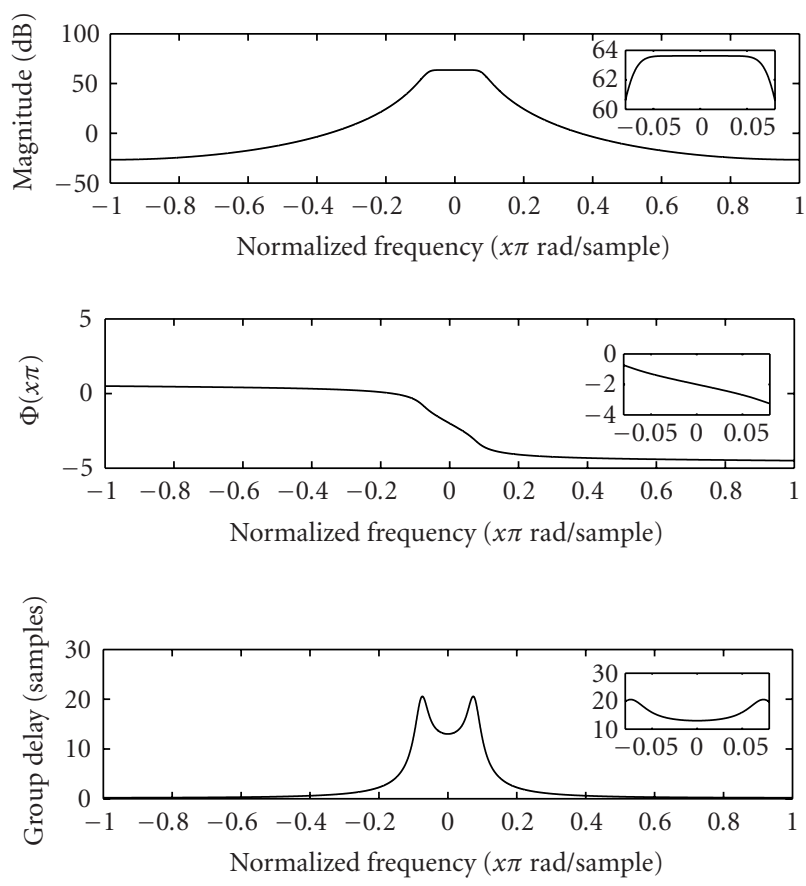

FIGURE 8: Frequency response and group delay characteristic of 5thorder AR filter.

TABLE 1: Design values for a 5th-order AR lattice filter.

\begin{tabular}{ccccccc}
\hline & $n=0$ & 1 & 2 & 3 & 4 & 5 \\
\hline$\kappa_{n}$ & 0.7336 & 0.1416 & 0.0357 & 0.0198 & 0.0232 & 0.2488 \\
$\phi_{n}$ & - & 0 & 0 & 0 & 0 & 0 \\
\hline
\end{tabular}

The frequency response and the group delay characteristic of this filter are presented in Figure 8 showing that the designed filter's response matches with the ideal prototype response of Figure 3 for nonlinear phase shift. The magnitude response is maximally flat as desired. Also, even though most of the group delay is pushed towards the passband edges, the group delay and magnitude response does not have ripples and hence bistability is largely avoided.
This discrete filter is then mapped onto the optical AR lattice architecture of Figure 6. Table 1 shows the coupling ratios and phase values thus obtained for each stage of the optical filter.

The linear response of the synthesized optical filter is the same as that of the discrete filter for low input intensity. The nonlinear phase shift response of the AR filter is shown in Figure 9 as a function of the normalized input intensity $n_{2} I_{\text {in }}$, where $n_{2}$ is the nonlinear coefficient of the underlying material and $I_{\text {in }}$ is the input intensity. As can be seen from the figure, a $\pi$ radian phase change is obtained at $n_{2} I_{\pi}=9.0 \times 10^{-5}$ and the transmission ratio at this input intensity is 0.66 . The nonlinear response is also plotted for incident frequencies at $v_{m} \pm \delta \nu / 4$ where $\nu_{m}$ is the center frequency. Because of the flat magnitude response in the filter's linear response, the nonlinear phase response (up to a $\pi$ phase shift) is weakly sensitive to frequency within the passband of the filter, as shown, allowing for a broadband nonlinearity. Also plotted for comparison is the phase shift produced by the underlying material of length $L=k_{\mathrm{gd}} c / n \sim 0.65 \mathrm{~mm}$, which gives the same group delay as that of the AR lattice architecture. The nonlinear phase shift produced by the designed AR filter is 5 times better than that of the bulk material.

The allowable amount of parameter error is an important information for fabrication. Random errors were added to each of the design parameters, that is, the coupling ratios and the phase values, and the nonlinear response was obtained to determine the parameter sensitivity. The allowable errors below which the nonlinear response is within $10 \%$ of the original value are $\pm 0.001 \pi$ for $\kappa_{r n}$, and $\pm 0.003 \pi$ for $\phi_{r n}$. A detailed sensitivity analysis is presented in [24].

\subsection{Improving the nonlinear phase shift response}

The nonlinear phase shift response improves upon increasing the group delay. This is because high group delay implies steeper phase response which results in greater nonlinear phase shift as the frequency response red shifts upon increasing input intensity. For a maximum phase discrete filter with no poles at the origin, the total phase change across the FSR is expressed by $\Phi_{\mathrm{ob}}+\Phi_{\mathrm{ib}}=2 \pi N_{z}$, where $\Phi_{\mathrm{ob}}$ is the outof-band phase, $\Phi_{\mathrm{ib}}$ is the in-band phase, and $N_{z}$ is the number of zeros in the discrete filter. This simple analysis shows 

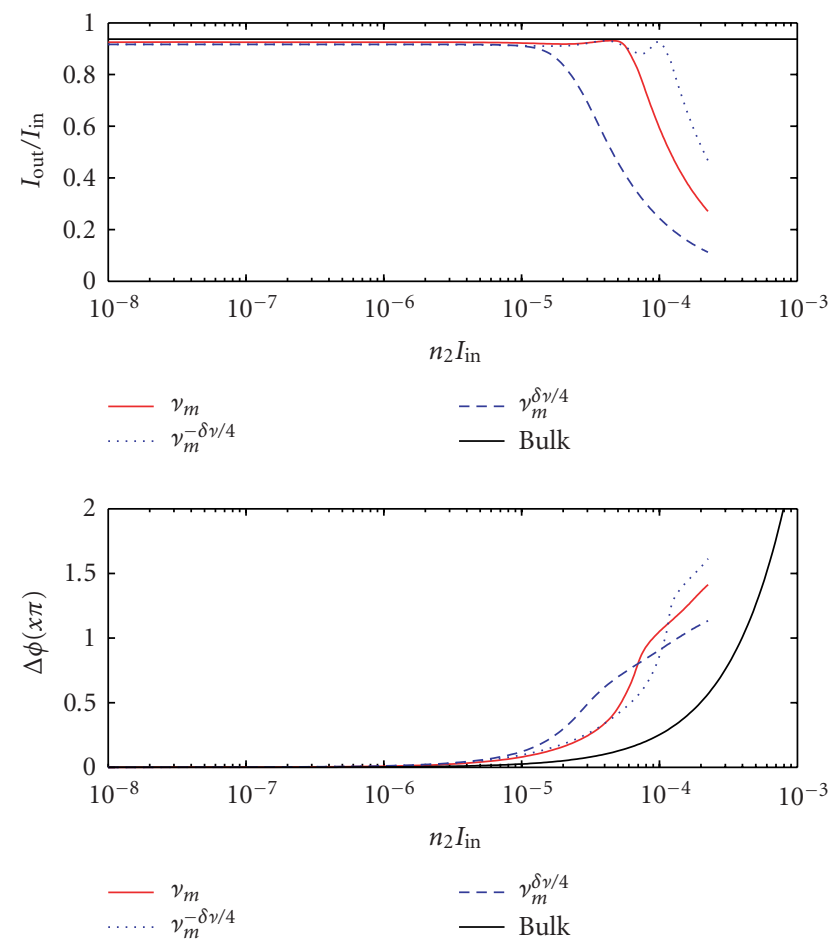

FIGURE 9: Nonlinear response vsersus incident intensity $n_{2} I_{\text {in }}$.

that there are two means to increase the group delay (and hence, the nonlinear response) within the passband:

(1) increase the in-band phase change $\Phi_{\mathrm{ib}}$, and/or

(2) increase the filter order.

In general, the bandwidth, $\delta v$ (along with the FSR) should be a quantity chosen at the outset to match a specific application. For example, if the desired application were to produce a phase shift on a single channel of a DWDM system, then $\delta v \sim \delta v_{\mathrm{ch}}$ and FSR $\sim N_{\mathrm{ch}} \delta v_{\mathrm{ch}}$, where $\delta v_{\mathrm{ch}}$ is the channel spacing and $N_{\mathrm{ch}}$ is the number of channels.

Since AR filters are designed using the generalized Butterworth filter design, we do not have control over the in-band phase to increase the group delay. We increase the group delay by increasing the filter order, that is, the number of stages in the architecture, which in turn increases the total phase as well as the in-band phase. Figure 10 plots $n_{2} I_{\pi}$ as a function of the group delay where the group delay is increased by increasing the filter order while keeping the bandwidth constant. The quantity $n_{2} I_{\pi}$ scales as $1 / k_{\mathrm{gd}}^{2.72}$ and is given by $n_{2} I_{\pi}=19.55 \times 10^{-4} k_{\mathrm{gd}}^{-2.72}$. The scaling of $n_{2} I_{\pi}$ with group delay is not an accurate representation of the initial design of the filter because by the time a $\pi$ radian nonlinear phase shift is obtained, the filter characteristics change (i.e., the new filter function is no longer just a shifted version of the initial function as assumed in the weak perturbation limit) because of increasing input intensity. Hence $n_{2} I_{\pi / 4}$ is plotted as a function of group delay and is shown in Figure 10 . The quantity $n_{2} I_{\pi / 4}$ scales as $1 / k_{\mathrm{gd}}^{0.82}$ and is given by $n_{2} I_{\pi / 4}=12.46 \times 10^{-5} k_{\mathrm{gd}}^{-0.82}$. This implies that in principle,

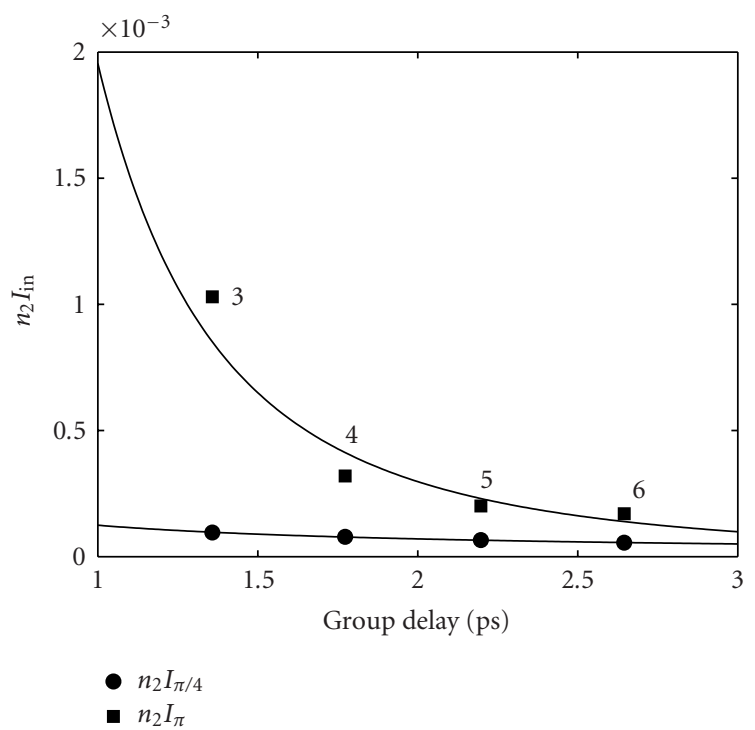

FIGURE 10: Improving nonlinear response by increasing the number of stages and keeping $\mathrm{BW}=0.12 \mathrm{FSR}$.

TABLE 2: Improving nonlinear response by increasing the AR filter order with BW $=0.12$ FSR.

\begin{tabular}{cccc}
\hline Filter order & Group delay $(\mathrm{ps})$ & $n_{2} I_{\pi}$ & $n_{2} I_{\pi / 4}$ \\
\hline 3 & 1.36 & $1.03 \times 10^{-3}$ & $9.59 \times 10^{-5}$ \\
4 & 1.77 & $3.20 \times 10^{-4}$ & $7.89 \times 10^{-5}$ \\
5 & 2.19 & $2.01 \times 10^{-4}$ & $6.56 \times 10^{-5}$ \\
6 & 2.64 & $1.70 \times 10^{-4}$ & $5.54 \times 10^{-5}$ \\
\hline
\end{tabular}

the nonlinear response can be improved while maintaining constant bandwidth by using higher-order filters. The filter order, group delay, $n_{2} I_{\pi}$, and $n_{2} I_{\pi / 4}$ are shown in Table 2 for a bandwidth of 0.12 FSR.

\section{EXAMPLE AND EVALUATION OF ARMA LATTICE FILTERS}

\subsection{Design and synthesis example}

In this section, we design an optical ARMA lattice filter and simulate it to obtain the nonlinear phase shift response. The filter is synthesized by designing discrete filters according to the description in Section 6.2 and then using the mapping algorithm derived by Jinguji [22]. The circumference of each microring in the ARMA lattice architecture is chosen as the unit delay length and is equal to $50 \mu \mathrm{m}$. The center frequency corresponds to a wavelength of $500 \mathrm{~nm}$.

A maximum phase ARMA filter with four zeros and four poles is designed using the procedure discussed in Section 6.2. The filter bandwidth is set to be $0.05 \pi$ in the fundamental range $-\pi \leq \omega \leq \pi$. $4 \pi$ out of the total $8 \pi$ phase change is allocated to the out-of-band phase change to maintain flat magnitude and linear phase response. Passband ripple is less than $0.1 \mathrm{~dB}$ and the stop-band magnitude is $18 \mathrm{~dB}$. 

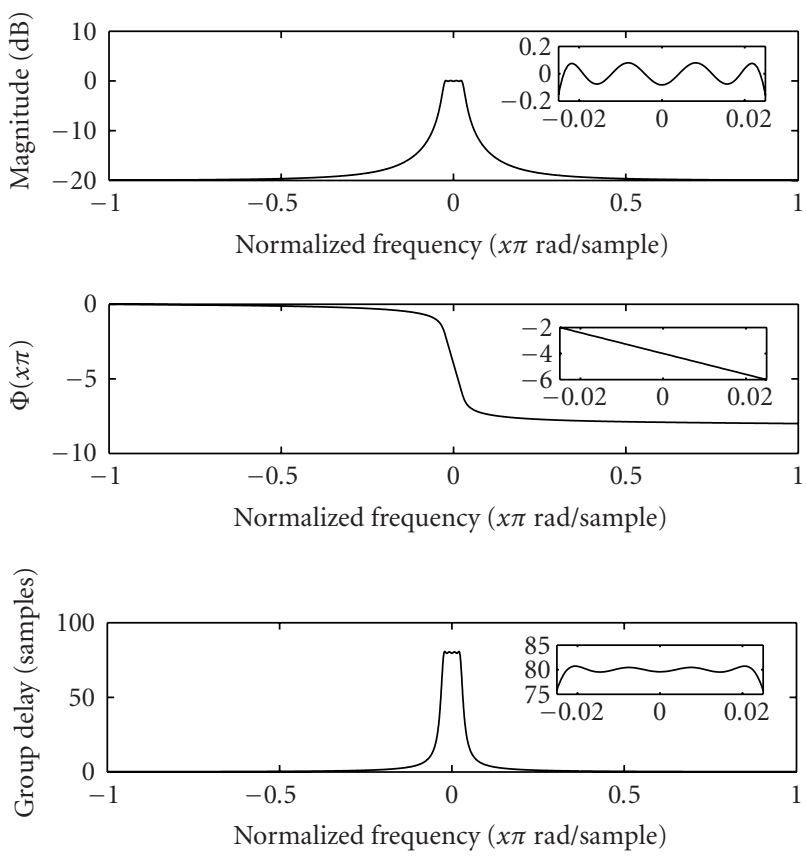

FIGURE 11: Frequency response and group delay characteristic of a 4th-order real ARMA filter.

TABLE 3: Design values for a 4th-order real ARMA lattice filter.

\begin{tabular}{cccccc}
\hline & $n=0$ & 1 & 2 & 3 & 4 \\
\hline$k_{t n}$ & 0.1555 & 0.5513 & 0.5289 & 0.1733 & 0.9418 \\
$\phi_{t n}$ & - & 2.9754 & -1.4868 & 1.3928 & 2.0702 \\
$k_{r n}$ & - & 0.0594 & 0.0594 & 0.0784 & 0.0784 \\
$\phi_{r n}$ & - & 0.0771 & -0.0771 & 0.0267 & -0.0267 \\
\hline
\end{tabular}

Assuming the loss in the material to be $1 \mathrm{~cm}^{-1}$, the obtained filter transfer function is

$$
\begin{aligned}
& \frac{N(z)}{D(z)} \\
& =\frac{0.0656-0.3176 z^{-1}+0.5661 z^{-2}-0.4424 z^{-3}+0.1283 z^{-4}}{1.0000-3.8531 z^{-1}+5.5736 z^{-2}-3.5872 z^{-3}+0.8667 z^{-4}} .
\end{aligned}
$$

The frequency response and the group delay characteristic of this filter are shown in Figure 11 showing that the designed filter's response matches with the ideal prototype response of Figure 3 for the nonlinear phase shift.

This discrete filter is then mapped onto the optical ARMA lattice architecture of Figure 7. Table 3 shows the coupling ratios and phase values thus obtained for each stage of the optical filter.

The linear response of the synthesized optical filter is the same as that of the discrete filter for low input intensity. The nonlinear phase shift response of the ARMA filter is shown in Figure 12 as a function of the normalized input intensity $n_{2} I_{\text {in }}$, where $n_{2}$ is the nonlinear coefficient of the underlying material and $I_{\text {in }}$ is the input intensity. A $\pi$ radian phase change is obtained at $n_{2} I_{\pi}=3.3 \times 10^{-6}$ and the transmission
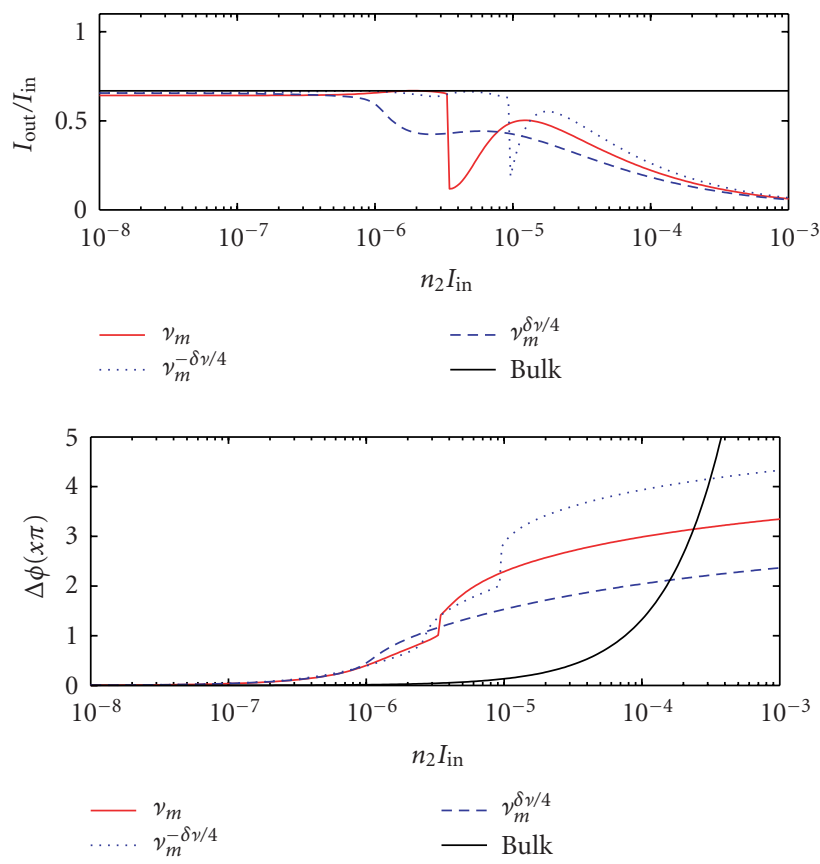

FIGURE 12: Nonlinear response versus incident intensity $n_{2} I_{\text {in }}$.

ratio at this input intensity is 0.65 . The nonlinear response is also plotted for incident frequencies at $v_{m} \pm \delta \nu / 4$ where $v_{m}$ is the center frequency. As in the case of AR filter, the flat magnitude response in the filter's linear response allows for a broadband nonlinearity. Also plotted for comparison is the phase shift produced by the underlying material of length $L=k_{\mathrm{gd}} c / n \sim 4 \mathrm{~mm}$, which gives the same group delay as that of the ARMA lattice architecture. The nonlinear phase shift produced by the filter is 19 times better than the bulk material [25]. The nonlinear phase shift enhancement over bulk material is larger in the case of ARMA filters because of two reasons. (1) The total phase change in the case of ARMA filters is twice that of AR filters for equal number of stages. This results in higher group delay in the case of ARMA filters and hence better nonlinear phase shift response. (2) The group delay in case of AR filters is pushed towards the passband edges and hence, lower group delay at center frequency results in lower nonlinear phase-shift enhancement.

As in the case of AR filters, random errors were added to each of the design parameters, and the nonlinear response was obtained to determine the parameter sensitivity. The allowable errors below which the nonlinear response is within $10 \%$ of the original value are $\pm 0.001 \pi$ for $\kappa_{r n}, \pm 0.001 \pi$ for $\phi_{r n}, \pm 0.01 \pi$ for $\kappa_{t n}$, and $\pm 0.01 \pi$ for $\phi_{t n}$. A detailed sensitivity analysis is presented in [24].

\subsection{Improving the nonlinear phase shift response}

Similar to AR filters, the nonlinear phase shift response improves upon increasing the group delay and two means to increase the group delay (aside from decreasing passband width) are to either increase the in-band phase change $\Phi_{i b}$, and/or increase the filter order. 


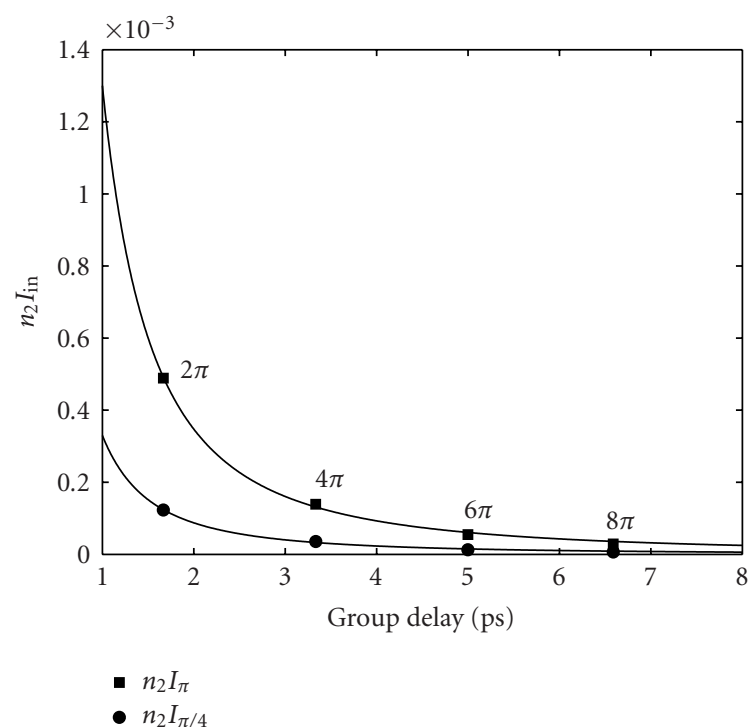

FIGURE 13: Improving nonlinear response by increasing the in-band phase for a 6th-order ARMA filter with $\mathrm{BW}=0.15 \mathrm{FSR}$.

TABLE 4: Improving nonlinear response by increasing the in-band phase for a 6 th-order ARMA filter with $\mathrm{BW}=0.15 \mathrm{FSR}$.

\begin{tabular}{cccc}
\hline In-band phase $\Phi_{\mathrm{ib}}$ & Group delay $(\mathrm{ps})$ & $n_{2} I_{\pi}$ & $n_{2} I_{\pi / 4}$ \\
\hline $2 \pi$ & 1.67 & $4.89 \times 10^{-4}$ & $1.23 \times 10^{-4}$ \\
$4 \pi$ & 3.34 & $1.39 \times 10^{-4}$ & $3.55 \times 10^{-5}$ \\
$6 \pi$ & 4.99 & $5.49 \times 10^{-5}$ & $1.30 \times 10^{-5}$ \\
$8 \pi$ & 6.59 & $2.90 \times 10^{-5}$ & $6.51 \times 10^{-6}$ \\
\hline
\end{tabular}

For a chosen bandwidth and fixed filter order, the first approach results in a trade-off between retaining the full phase within the band and in-band ripple (there is also a trade-off between $\Phi_{\mathrm{ib}}$ and rejection ratio, but, unlike for true bandpass filters, here we are not concerned with having high rejection). Therefore, a certain amount of the total phase change needs to be allocated to $\Phi_{\mathrm{ob}}$ in order to reduce ripple. Figure 13 plots $n_{2} I_{\text {in }}$ as a function of the group delay where group delay is increased by increasing the in-band phase in a 6thorder ARMA lattice filter while keeping a constant bandwidth of $0.15 \mathrm{FSR}$. The quantity $n_{2} I_{\pi}$ scales as $1 / k_{\mathrm{gd}}^{1.90}$ and is given by $n_{2} I_{\pi}=1.30 \times 10^{-3} k_{\text {gd }}^{-1.90}$. The quantity $n_{2} I_{\pi / 4}$ scales as $1 / k_{\mathrm{gd}}^{1.92}$ and is given by $n_{2} I_{\pi / 4}=3.30 \times 10^{-4} k_{\mathrm{gd}}^{-1.92}$. The in-band phase, group delay, $n_{2} I_{\pi}$, and $n_{2} I_{\pi / 4}$ are shown in Table 4.

The second approach increases the group delay by increasing the filter order, that is, the number of stages in the architecture, which in turn increase the total phase as well as the in-band phase. Figure 14 plots $n_{2} I_{\text {in }}$ as a function of the group delay where the group delay is increased by increasing the filter order while keeping the bandwidth and

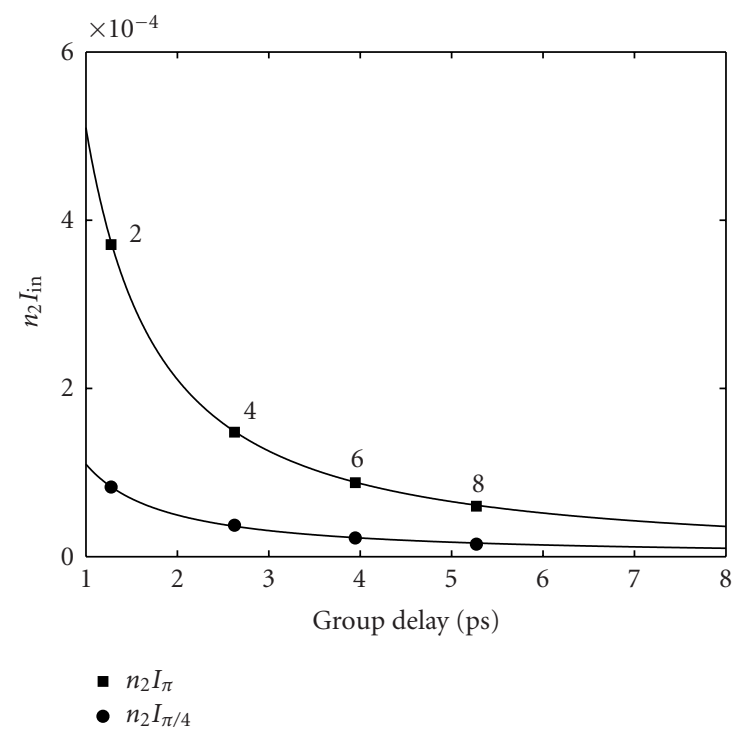

FIGURE 14: Improving nonlinear response by increasing the number of stages and keeping BW $=0.19 \mathrm{FSR}, \Phi_{\mathrm{ib}} / \Phi_{\mathrm{ob}}=0.5$.

TABLE 5: Improving nonlinear response by increasing the ARMA filter order with $\mathrm{BW}=0.19 \mathrm{FSR}$, in-band to out-band phase ratio $=$ 0.5 .

\begin{tabular}{cccc}
\hline Filter order & Group delay $(\mathrm{ps})$ & $n_{2} I_{\pi}$ & $n_{2} I_{\pi / 4}$ \\
\hline 2 & 1.27 & $3.71 \times 10^{-4}$ & $8.27 \times 10^{-5}$ \\
4 & 2.62 & $1.48 \times 10^{-4}$ & $3.73 \times 10^{-5}$ \\
6 & 3.95 & $8.80 \times 10^{-5}$ & $2.23 \times 10^{-5}$ \\
8 & 5.27 & $6.01 \times 10^{-5}$ & $1.50 \times 10^{-5}$ \\
\hline
\end{tabular}

the $\Phi_{\mathrm{ib}} / \Phi_{\mathrm{ob}}$ ratio constant. The quantity $n_{2} I_{\pi}$ scales as $1 / k_{\mathrm{gd}}^{1.28}$ and is given by $n_{2} I_{\pi}=5.10 \times 10^{-4} k_{\mathrm{gd}}^{-1.28}$. The quantity $n_{2} I_{\pi / 4}$ scales as $1 / k_{g d}^{1.15}$ and is given by $n_{2} I_{\pi / 4}=1.10 \times 10^{-4} k_{\mathrm{gd}}^{-1.15}$. This implies that in principle, the nonlinear response can be improved while maintaining constant bandwidth by using higher-order filters. The filter order, group delay, $n_{2} I_{\pi}$, and $n_{2} I_{\pi / 4}$ are shown in Table 5 for a bandwidth of 0.19 FSR and the in-band to out-band phase ratio of 0.5 .

\section{CONCLUSIONS}

In this paper, we have proposed that a discrete-time signal processing approach can be used to design nonlinear optical devices by treating the desired nonlinear response in the weak perturbation limit as a linear discrete filter. This provides a systematic and intuitive method for the design of nonlinear optical devices. We have demonstrated this approach by designing AR and ARMA filters to obtain a nonlinear phase shift response. This approach can be used for designing optical devices for various other nonlinear processes as well. 


\section{REFERENCES}

[1] S. Blair, J. E. Heebner, and R. W. Boyd, "Beyond the absorption-limited nonlinear phase shift with microring resonators," Optics Letters, vol. 27, no. 5, pp. 357-359, 2002.

[2] C. K. Madsen and J. H. Zhao, Optical Filter Design and Analysis: A Signal Processing Approach, Wiley, New York, NY, USA, 1999.

[3] T. A. Ibrahim, V. Van, and P.-T. Ho, "All-optical time-division demultiplexing and spatial pulse routing with a GaAs AlGaAs microring resonator," Optics Letters, vol. 27, no. 10, pp. 803805, 2002.

[4] M. Soljacic, C. Luo, J. D. Joannopoulos, and S. Fan, "Nonlinear photonic crystal microcavities for optical integration," Optics Letters, vol. 28, no. 8, pp. 637-639, 2003.

[5] S. Blair, Optical soliton-based logic gates, Ph.D. dissertation, University of Colorado, Boulder, Colo, USA, 1998.

[6] M. Soljacic, S. G. Johnson, S. Fan, et al., "Photonic-crystal slow-light enhancement of nonlinear phase sensitivity," Journal of the Optical Society of America B, vol. 19, no. 9, pp. 20522059, 2002.

[7] Y. Chen and S. Blair, "Nonlinear phase shift of cascaded microring resonators," Journal of Optical Society of America B, vol. 20, no. 10, pp. 2125-2132, 2003.

[8] W. Nakagawa, R.-C. Tyan, and Y. Fainman, "Analysis of enhanced second-harmonic generation in periodic nanostructures using modified rigorous coupled-wave analysis in the undepleted-pump approximation," Journal of the Optical Society of America A, vol. 19, no. 9, pp. 1919-1928, 2002.

[9] P. P. Absil, J. V. Hryniewicz, B. E. Little, et al., "Wavelength conversion in GaAs micro-ring resonators," Optics Letters, vol. 25, no. 8, pp. 554-556, 2000.

[10] A. Melloni, F. Morichetti, and M. Martinelli, "Optical slow wave structures," Optics and Photonics News, vol. 14, no. 11, pp. 44-48, 2003.

[11] A. Hasegawa and Y. Kodama, "Signal transmission by optical solitons in monomode fiber," Proc. IEEE, vol. 69, no. 9, pp. 1145-1150, 1981.

[12] A. Melloni, F. Morichetti, and M. Martinelli, "Linear and nonlinear pulse propagation in coupled resonator slow-wave optical structures," Optical and Quantum Electronics, vol. 35, no. 4-5, pp. 365-379, 2003.

[13] J. E. Heebner and R. W. Boyd, "SCISSOR solitons and other novel propagation effects in microresonator-modified waveguides," Journal of the Optical Society of America B, vol. 19, no. 4, pp. 722-731, 2002.

[14] D. A. B. Miller, S. D. Smith, and A. Johnston, "Optical bistability and signal amplification in a semiconductor crystal: applications of new low-power nonlinear effects in InSb," Applied Physics Letters, vol. 35, no. 9, pp. 658-660, 1979.

[15] H. M. Gibbs, Optical Bistability: Controlling Light with Light, Academic Press, Orlando, Fla, USA, 1985.

[16] M. Soljacic, M. Ibanescu, S. G. Johnson, Y. Fink, and J. D. Joannopoulos, "Optimal bistable switching in nonlinear photonic crystals," Physical Review E, vol. 66, no. 5, pp. 055601-1055601-4, 2002.

[17] I. W. Selesnick and C. S. Burrus, "Generalized digital butterworth filter design," in Proc. IEEE Int. Conf. Acoustics, Speech, Signal Processing (ICASSP '99), vol. 3, pp. 90-95, Phoenix, Ariz, USA, May 1999.

[18] P. P. Vaidyanathan, Multirate Systems and Filter Banks, Prentice Hall, Englewood Cliffs, NJ, USA, 1993.

[19] B. Farhang-Boroujeny, Adaptive Filters: Theory and Applications, John Wiley \& Sons, Chichester, UK, 1998.
[20] E. C. Levi, “Complex-curve fitting," IRE Transactions on Automatic Control, vol. 4, pp. 37-44, 1959.

[21] C. K. Madsen and J. H. Zhao, "A general planar waveguide autoregressive optical filter," J. Lightwave Technol., vol. 14, no. 3, pp. 437-447, 1996.

[22] K. Jinguji, "Synthesis of coherent two-port optical delay line circuits with ring waveguides," J. Lightwave Technol., vol. 14, no. 8, pp. 1882-1898, 1996.

[23] Y. Chen, Nonlinear optical process enhancement by artificial resonant structures, Ph.D. dissertation, University of Utah, Salt Lake City, Utah, USA, 2004.

[24] G. Pasrija, "Discrete-time signal processing approach to the design of nonlinear optical devices," M.S. thesis, University of Utah, Salt Lake City, Utah, USA, 2004.

[25] Y. Chen, G. Pasrija, B. Farhang-Boroujeny, and S. Blair, "Engineering the nonlinear phase shift," Optics Letters, vol. 28, no. 20, pp. 1945-1947, 2003.

Geeta Pasrija received her M.S. degree in electrical engineering from the University of Utah in 2004. Currently, she is working at SR Technologies Inc., and is involved in the design of a software-defined radio for satellite communications.

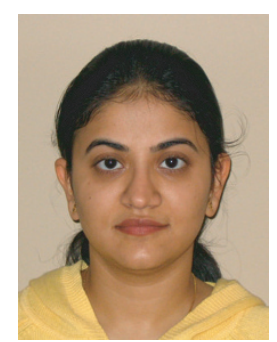

Yan Chen received her Ph.D. degree in electrical engineering from the University of Utah in 2004. From 1997 to 1999, she worked at Optical Memory National Engineering Research Center China, as a Research Assistant and Lecturer. Currently, she is working in the area of semiconductor lithography software development for Timbre Technologies Inc.

Behrouz Farhang-Boroujeny received his Ph.D. degree from Imperial College, University of London, UK, in 1981. From 1981 to 1989 he was at Isfahan University of Technology, Isfahan, Iran. From 1989 to 2000 he was at the National University of Singapore. Since August 2000, he has been with the University of Utah. He is an expert in the general area of signal processing. His current scientific interests are in adaptive fil-

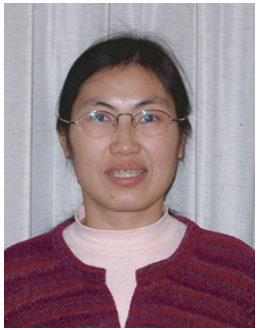
ters, multicarrier communications, detection techniques for spacetime coded systems, and signal processing applications to optical devices.

Steve Blair received his $\mathrm{Ph} . \mathrm{D}$. degree from the University of Colorado at Boulder in 1998. He has been an Assistant Professor in the Electrical and Computer Engineering Department at the University of Utah since then. His research interests include slowlight nonlinear optics, nanoscale photonics, and real-time molecular detection arrays.

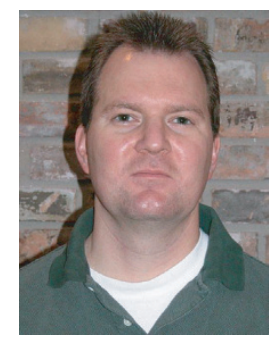

